\title{
Peritoneal Serous Papillary Adenocarcinoma: Report of Four Cases
}

\author{
Takeshi MinAmi, Koji NAKATAni, Shinya Kondo, Shuji KAnAYAma and Takahiro TsuJimurA*
}

\begin{abstract}
We report four cases of peritoneal serous papillary adenocarcinoma (PSPC), a rare disease; all patients had ascites and high levels of serum CA125. Clinical and radiological examinations could not differentiate the disease from peritoneal metastatic tumors and mesothelioma, and histopathological analysis including immunochemistry on the specimen obtained at laparotomy or laparoscopy was necessary for the diagnosis. One patient lived for 58 months with cytoreductive surgery and chemotherapy, and another is still living after 20 months by chemotherapy alone. In patients with peritoneal tumors of unknown origin and a high level of serum CA125, taking PSPC into consideration in the differential diagnosis, histopathological examination should be performed. (Internal Medicine 44: 944-948, 2005)
\end{abstract}

Key words: peritoneum, chemotherapy, immunohistochemistry

\section{Introduction}

Peritoneal serous papillary adenocarcinoma (PSPC) is a rare primary peritoneal neoplasm, rising exclusively in menopausal and post-menopausal women. This disease is histologically similar to ovarian serous papillary adenocarcinoma, but is characteristic of the tumor cells mainly disseminated on the peritoneum with almost normal-sized ovaries. This disease is clinically difficult to differentiate from peritoneal mesothelioma and metastatic tumors of the peritoneum, and the diagnosis is made at the time of laparotomy or autopsy in most cases. Here, we have reported four cases of PSPC found at the Sumitomo hospital and discussed the clinical, radiological, and immunohistochemical appearance of the disease.

\author{
Case Reports
}

\section{Case 1}

A 71-year-old woman visited Sumitomo hospital complaining of abdominal distention and was admitted in February 1989 because of massive ascites. From laboratory data, anemia $(\mathrm{Hb} 9.2 \mathrm{mg} / \mathrm{dl})$ and a high level of serum CA125 (10,330 U/ml) were seen. Abdominal CT revealed a poorly defined hazy mass in the anterior portion of the peritoneal cavity with retroperitoneal lymphadenopathy, omental caking appearance, and ascites. Gynecological survey, and endoscopic examinations of the upper gastrointestinal tract and colon did not reveal neoplastic lesions. Because adenocarcinoma cells were found in the ascites specimen on cytologic examination, intraperitoneal injection of cisplatin was started. Then, the ascites and the serum CA125 levels decreased. On the 66th hospital day, she complained of sudden abdominal pain, and free air was intraperitonally observed on abdominal X-P. Laparotomy revealed fecal fluid filling the abdomen, an omental tumor obstructing the transverse colon, perforation at the cecum, and multiple nodules on the peritoneum. No abnormalities were found in other organs including the ovaries and uterus. The tumor and the affected transverse colon were resected. Additional chemotherapy could not be performed because of her poor condition caused by bacterial peritonitis. She died 2 months after the surgery.

\section{Case 2}

A 44-year-old woman was admitted because of abdominal distention and ascites in October 1989. A serum CA125 level was elevated to $3,590 \mathrm{U} / \mathrm{ml}$. Cytologic examination of the ascites showed adenocarcinoma cells. Abdominal CT revealed an omental tumor, omental caking appearance, and ascites, but no other abnormalities (Fig. 1A). Exploratory laparotomy revealed the omental tumor involving the colon and multiple nodules on the peritoneum, and tumorous lesions were also seen on the surface of the bilateral ovaries

From the Department of Gastroenterology and *the Department of Pathology, Sumitomo Hospital, Osaka

Received for publication November 22, 2004; Accepted for publication April 11, 2005

Reprint requests should be addressed to Dr. Takeshi Minami, Department of Gastroenterology, Sumitomo Hospital, 5-3-20 Nakanoshima, Kita-ku, Osaka $530-0005$ 

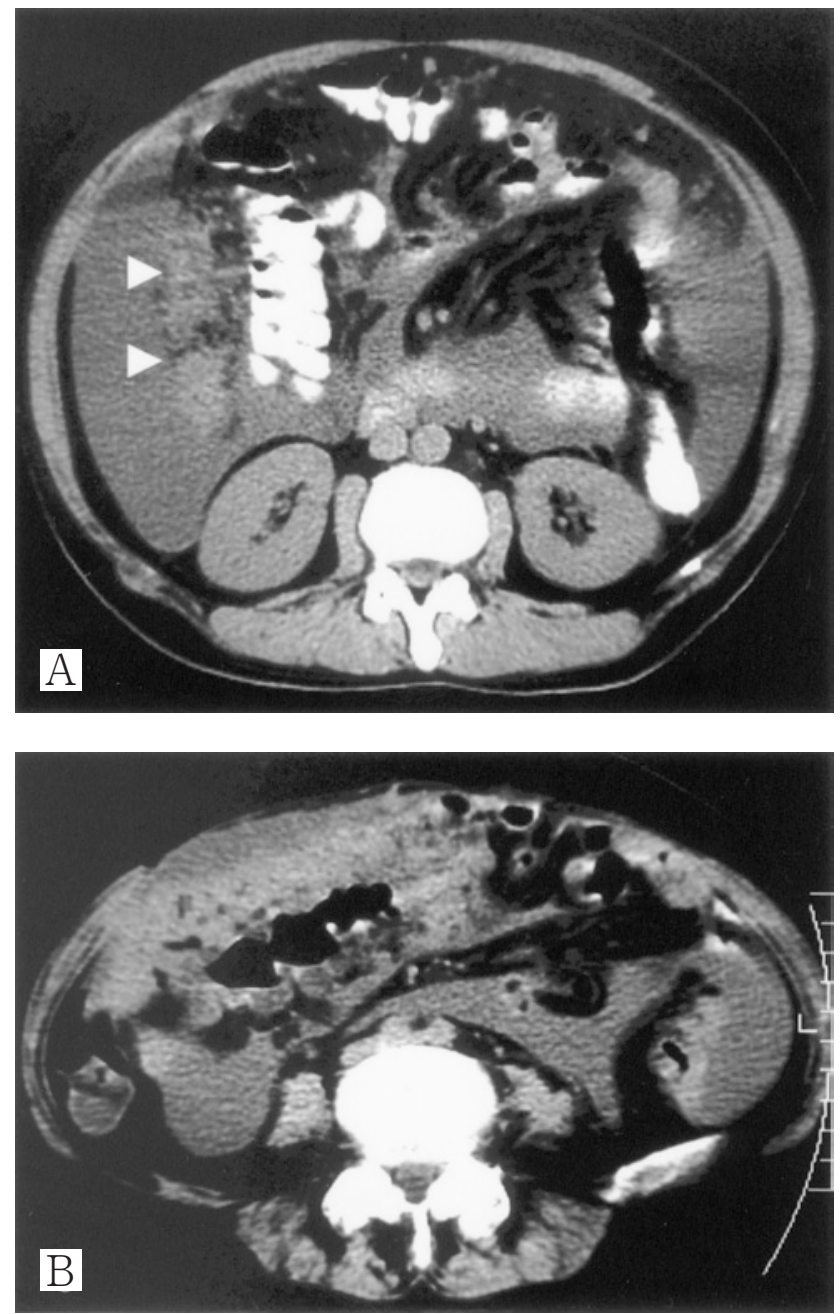

Figure 1. (A) Abdominal CT at the level of kidney in case 2 shows a poorly demarcated mass (arrowhead) surrounded by fat density adjacent to the colon and ascites. (B) CT of the lower abdomen in case 3 reveals a smudgy, large omental tumor attached to the transverse colon and ascites.

and fallopian tubes, but no abnormalities were found in other organs. Partial resection of the omental tumor was performed, and intravenous injection of cisplatin was started. The amount of the ascites and the serum CA125 levels were temporally decreased, but the patient's condition gradually worsened. She died 6 months after admission.

\section{Case 3}

A 59-year-old woman with abdominal distention was admitted in August 1993 because of an abdominal mass and ascites. Her serum CA125 level was 11,100 U/ml. Adenocarcinoma cells were found in the ascitic fluid. Abdominal CT showed a smudgy, huge mass in front of the transverse colon and ascites (Fig. 1B). Double-contrast enema showed irregularity on the upper aspect of the transverse colon. Upper gastrointestinal endoscopic examination found no

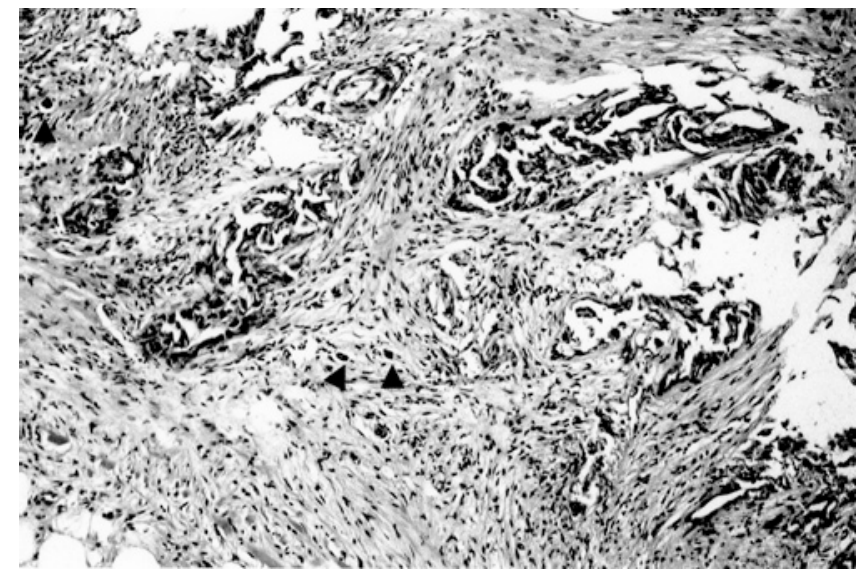

Figure 2. Light microscopic features of the tumor obtained from case 4, showing a tubulopapillary structure of the tumor cells with nuclear atypism and psammoma bodies (arrowhead) (HE stain, $\times 30)$.

abnormalities. At laparotomy, a large omental tumor involving the transverse colon, multiple nodules scattered on the peritoneum and on the surface of the ovaries were found. Resection of the tumor and the affected transverse colon with bilateral adnexectomy was performed, and a peritoneal infusion catheter was implanted. Intravenous infusion of carboplatin and intraperitoneal infusion of cisplatin were begun. The residual intraperitoneal tumors and ascites then disappeared, and the serum CA125 levels decreased to within the normal limit. She died 58 months after the surgery because of septic shock. No recurrence of the tumor was observed during her life.

\section{Case 4}

A 58-year-old woman complaining of abdominal distention was admitted in January 2003 because of massive ascites and slight right pleural effusion. She had right adnexectomy 29 years before because of an ovarian cyst. A serum CA125 level was $693 \mathrm{U} / \mathrm{ml}$. Cytologic examination of the ascites and pleural effusion showed similar adenocarcinoma cells. Abdominal CT and MRI demonstrated thickening of the omentum, omental caking appearance and ascites, but no abnormalities were found in other organs including the left ovary and uterus. A gynecological survey including ultrasonography, chest $\mathrm{CT}$, endoscopic examinations of the upper gastrointestinal tract and colon did not reveal neoplastic lesions. Laparoscopic examination revealed omental caking massively involving the small intestine and multiple small nodules on the peritoneum. No abnormalities were macroscopically found in the ovary. Because resection of the tumor was determined to be impossible, only biopsy of the tumor was performed. Intravenous injection of cisplatin was started. The intraperitoneal tumor was reduced, the ascites disappeared, and the serum CA125 levels decreased. In contrast, the right pleural effusion increased. Then, 
MINAMI et al

Table 1. CEA and CA19-9 Levels in Sera and Ascites, and Hyaluronic Acid Levels in Ascites of Our Four Cases

\begin{tabular}{cccccc}
\hline Case & s-CEA $(\mathrm{U} / \mathrm{l})$ & $\mathrm{s}-\mathrm{CA} 19-9(\mathrm{U} / \mathrm{ml})$ & $\mathrm{p}-\mathrm{CEA}(\mathrm{U} / \mathrm{l})$ & $\mathrm{p}-\mathrm{CA} 19-9(\mathrm{U} / \mathrm{ml})$ & $\mathrm{p}-\mathrm{HA}(\mathrm{ng} / \mathrm{ml})$ \\
\hline 1 & 0.8 & 10 & 0.6 & $<2$ & 60,000 \\
2 & 1.0 & 42 & 0.8 & 3.6 & 31,600 \\
3 & 0.5 & 8 & 0.1 & $<2$ & 48,200 \\
4 & 0.5 & $<2$ & 0.6 & $<2$ & 28,300 \\
\hline
\end{tabular}

s-: serum, p-: ascitic, HA: hyaluronic acid.

Table 2. Results of Immunohistochemical Examination of Our Four Cases

\begin{tabular}{cccccccccc}
\hline Case & CK & EMA & MOC-31 & Ber-EP4 & HBME-1 & Calretinin & CK5/6 & Vimentin & CA125 \\
\hline 1 & + & + & + & + & - & - & - & - & + \\
2 & + & + & + & + & - & - & - & - & + \\
3 & + & + & + & + & + & - & - & - & + \\
4 & + & + & - & + & + & - & - & - & + \\
\hline
\end{tabular}

CK: cytokeratin, EMA: epithelial membrane antigen, CK5/6: cytokeratin5/6.

intrathoracic injection of cisplatin and OK432 was performed, and the effusion decreased. She has now been alive for 20 months. For reference, the data of the serum CEA and CA19-9 levels, and the levels of CEA, CA19-9 and hyaluronic acid in the ascites of these four patients are shown in Table 1.

\section{Immunohistopathological findings}

Specimens of the tumors and organs were obtained at laparotomy (cases 1 and 3), at autopsy (case 2), and at laparoscopic examination (case 4). For light microscopic examination, these samples were fixed in $20 \%$ buffered formalin with phosphate and embedded in paraffin. Serial sections were prepared and stained with hematoxylin and eosin (HE) for histological examination. Periodic acid Schiff with diastase digestion (PAS-D) and alcian blue stains were also performed. Immunohistochemistry was performed using the avidin-biotin-peroxidase complex procedure (1). The primary antibodies used were cytokeratin (CK), epithelial membrane antigen (EMA), MOC-31, Ber-EP4, HBME-1, cytokeratin5/6, carletinin, vimentin, CA125, CEA, and CA19-9.

On histological examination, the tumor cells showed tubulopapillary structures with nuclear atypism, and many psammoma bodies were scattered within the tumor tissues in all the patients (Fig. 2). The tumors were diagnosed as poorly differentiated serous papillary adenocarcinoma in cases 1-3 and moderately differentiated in case 4 . In cases 2 and 3 , the tumors were macroscopically found on the surface of the bilateral ovaries. Microscopically, in case 2, tumorous lesions were found in the cortex with mild invasion of the underlying parenchyma and in case 3 , only in the cortex of the bilateral ovaries. In both of these cases, however, the tumor cells did not show an exophytic growth pattern from the ovarian surface which is a definitive feature of ovarian serous papillary adenocarcinoma. The mucosas of the resected transverse colons were free of the tumors in cases 1 and 3. The primary sites of the tumors except for the omentum were not found in the obtained specimens in cases 1-3. The tumor cells were positive with PAS-D technique, but negative for alcian blue stain in all four cases. The results of immunohistochemistry are summarized in Table 2. The tumor cells of all cases were positive for CK, EMA, Ber-EP4 (Fig. 3A), and CA125 (Fig. 3B), but negative for cytokeratin5/6, carletinin (Fig. 3C), vimentin, CEA, or CA19-9. In three (cases 1-3), the cells were positive for MOC-31, but negative in case 4 . In cases 3 and 4, the cells were positive for HBME-1, but in cases 1 and 2, the cells were negative.

\section{Discussion}

PSPC is one of the diseases included in normal-sized ovary syndrome proposed by Feuer et al (2). This disease clinically and pathologically resembles advanced ovarian serous papillary adenocarcinoma, but in PSPC, the ovaries are either almost normal or minimally involved, and carcinoma cells are mainly disseminated on the omentum and the peritoneum. These pathological characteristics were consistent with the tumors of our four cases. PSPC is a rare disease, and about 60 cases have been reported in Japan (3-11). According to these reports, all the patients were women aged 30-84 years. The most common symptoms were related to general abdominal discomfort including pain, distention or expansion and bloating, and the most common finding was ascites. In addition, in most cases, high levels of serum CA125 are reported. The definitive diagnosis by clinical and radiological examinations was impossible, and all cases were diagnosed by histopathological examination after laparotomy or autopsy. 

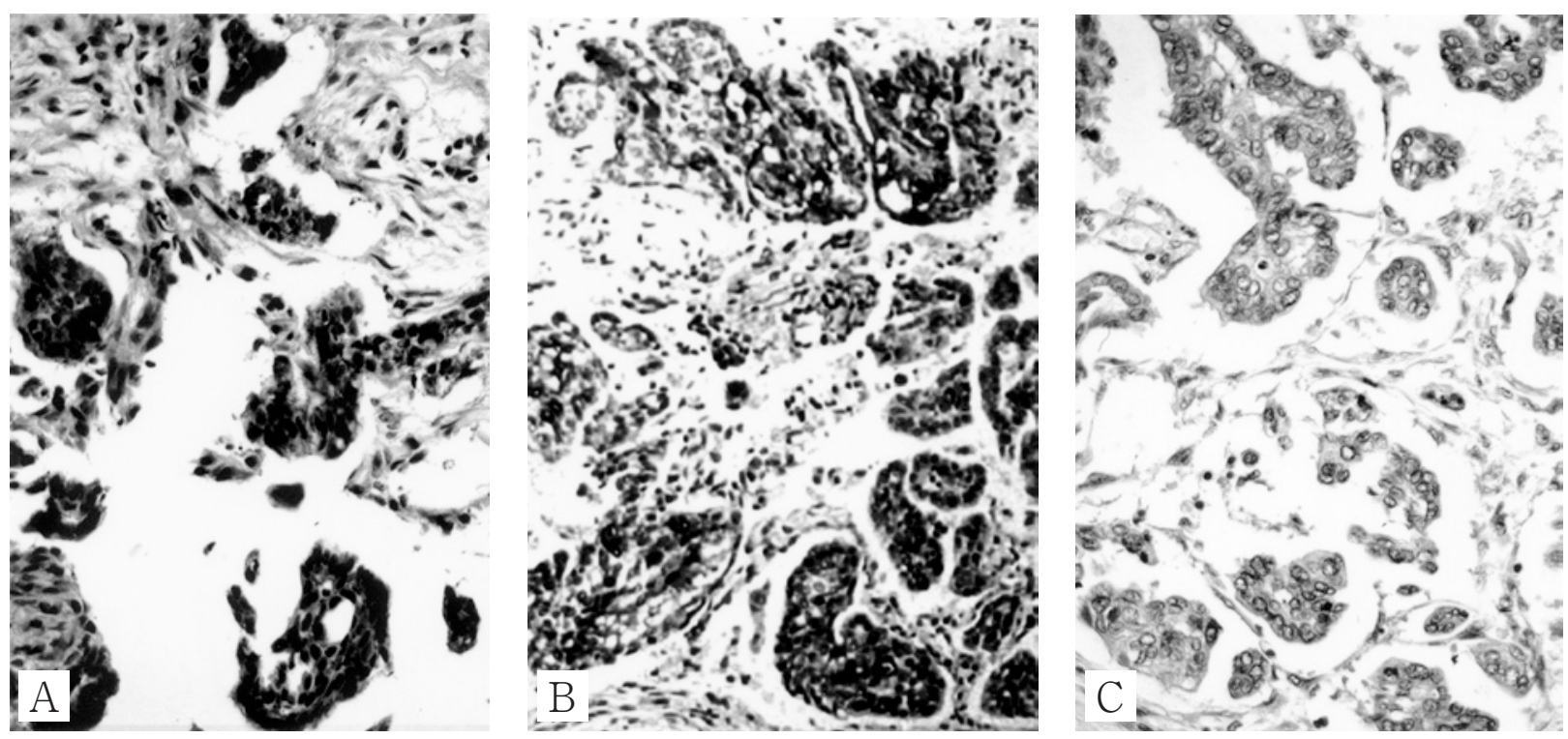

Figure 3. Immunohistochemical examination of case $1(\times 100)$. The tumor cells obtained from case 1 revealed positivity for Ber-EP4 (A) and CA125 (B), but negativity for carletinin (C).

In the present four cases, all the patients were also women aged 44-71 years and visited our hospital complaining of abdominal distention accompanied by massive ascites. In laboratory data, all the patients had high levels of serum CA125. Cytologic examination of the ascites was positive for adenocarcinoma cells, and all the patients were first suspected to have cancerous peritonitis which is often caused by metastatic peritoneal tumors. However, the primary sites could not be found by clinical examinations including gynecological survey, gastrointestinal endoscopy and ultrasonography. Abdominal CT showed omental caking in cases 1, 2, and 4 , a poorly demarcated, smudgy tumor in case 3 , and massive ascites in all cases without any identified primary sites or ovarian masses. These CT findings, however, are not specific to PSPC, but are also seen in other peritoneal disease. For example, ascites, omental caking, intra-abdominal masses, discrete nodules, and so on have been reported to be the characteristics of CT findings in metastatic peritoneal tumors and peritoneal mesothelioma (12-14). Therefore, the diagnosis of PSPC could not be made without histopathological examination including immunochemistry in our four cases.

Of our four cases, two were diagnosed at laparotomy, one at autopsy, and the other by laparoscopic examination. Previously, PSPC has been reported (15-17) to be difficult to differentiate from peritoneal mesothelioma and metastatic tumors of the peritoneum before surgery, and most cases are diagnosed at laparotomy or autopsy as in the present cases $1-3$. To our knowledge, there is only one report of a diagnosis by laparoscopic examination (18) as in our case 4 .

Here, on histological examination, many psammoma bodies and tubulopapillary structures were found in the tumors with HE stain obtained from all four patients. However, differential diagnosis of PSPC from mesothelioma is often difficult by only histological examination. On mucinous stain, the tumor cells of all our cases were positive for PAS$\mathrm{D}$, but negative for alcian blue. This showed that these cells produced diastase-resistant neutral mucins, but did not produce acid mucins. This finding suggested that the tumor cells had a character of glandular epithelium rather than that of mesothelial cells. On immunochemical analysis, all cases showed positivity for CK, EMA, Ber-EP4 as epithelial markers, and CA125. Three of the four cases showed positivity for MOC-31. In contrast, all cases were negative for cytokeratin $5 / 6$ and calretinin as mesothelial markers, and vimentin, but two of the four cases were positive for HBME1. HBME-1 is a mesothelial marker developed using a suspension of malignant mesothelioma cells, but this marker has been reported to show positivity for adenocarcinoma in about $50-60 \%$ (19-21). While, MOC-31 is an epithelial marker directed against a lung carcinoma cell line. Although its sensitivity for adenocarcinoma cells is relatively high, this marker has been reported to show negativity in about 6-10 $\%$ of these cells $(20,22)$. Taking these reports into account, we think that the results of our immunochemical examination were not necessarily inconsistent with the diagnosis of PSPC. In other words, immunochemical examination using multiple epithelial and mesothelial markers is thought to be useful for differentiating PSPC from mesothelioma. From the findings of the presence of many psammoma bodies on histopathological analysis, the results of mucinous stain, positivity of the tumor cells for multiple epithelial markers 
and CA125, but negativity for cytokeratin $5 / 6$, carletinin, and vimentin which have high sensitivity for mesothelioma (22, 23), we finally diagnosed all the patients as having PSPC.

In PSPC, the prognosis of the patients treated with chemotherapy alone, especially in those intensive intravenous chemotherapy cannot be done, is generally poor $(10,24)$. In contrast, mean survival periods of those treated with both cytoreductive surgery and intensive platinum-based chemotherapy have been reported to be 17-24 months (25-27). Here, one patient (case 3) lived for 58 months without recurrence of the tumor by optimal cytoreductive surgery together with intensive intravenous and intraperitoneal chemotherapy, and another (case 4) is still living for 20 months with intensive intravenous and intrathoracic chemotherapy. In contrast, the other two patients (cases 1 and 2) died within 6 months after admission. In case 1, only intraperitoneal chemotherapy before surgery was done, and additional chemotherapy could not be performed after the surgery because of the patient's poor condition caused by bacterial peritonitis secondary to perforation at the cecum. In case 2 , only a small amount of cisplatin could be intravenously given because of the patient's poor general condition and side effects. The fact that intensive intravenous chemotherapy could not be performed in these two patients may have led to the poor prognosis.

In conclusion, in patients, especially in women, with peritoneal tumors of unknown origin accompanied by elevated serum CA125 levels, taking PSPC into account, histopathological examination should be performed through laparoscopy or laparotomy. Because better prognosis can be possibly expected by platinum-based chemotherapy with or even without cytoreductive surgery in patients with PSPC than in those with peritoneal metastatic carcinomas and mesotheliomas.

\section{References}

1) Hsu SM, Raine L, Anger H. A comparative study of the peroxidaseantiperoxidase method and an avidin-biotin complex method for studying polypeptide hormones with radioimmunoassay antibodies. Am J Clin Pathol 75: 734-738, 1981.

2) Feuer GA, Shevchuk M, Calanog A. Normal sized ovary carcinoma syndrome. Obstet Gynecol 73: 786-792, 1989.

3) Toyonaga $\mathrm{T}$, Matsuo $\mathrm{K}$, Yamaguchi $\mathrm{H}$, et al. A case of serous papillary adenocarcinoma of the retroperitoneum. Shoukakigeka 23: 1325-1330, 2000 (in Japanese).

4) Kamiya S, Terasaki M, Okamoto Y, et al. A case of peritoneal serous papillary carcinoma. Nippon Rinsho Geka Gakkai Zassi 61: 13091314, 2000 (in Japanese).

5) Hasegawa K, Kamano N, Ogura T, et al. A case of extraovarian peritoneal serous papillary carcinoma. Jpn J Gastroenterol Surg 35: 17491753, 2002 (in Japanese).

6) Yamazaki T, Hatano H, Suzuki A, et al. Normal-sized ovary carcinoma syndrome: histopathological analysis of 14 cases. Nippon Sanka Fujinka Gakkai Zassi 47: 27-34, 1995 (in Japanese).

7) Onisi I, Kamata T, Hayashi H, et al. A case of peritoneal serous papillary adenocarcinoma. Jpn J Gastroenterol Surg 34: 1552-1555, 2001 (in Japanese).
8) Hara Y, Kareyama H, Sakai K, et al. Clinical analysis of peritoneal serous surface papillary carcinoma. Acta Obst Gynaec Jpn 52: 626630, 2000 (in Japanese)

9) Ueda K, Kawai T, Kobayashi Y, Usui Y, Higashi R, Nomura S. A case of serous papillary adenocarcinoma. Nippon Rinsho Geka Gakkai Zassi 64: 2905-2908, 2003 (in Japanese).

10) Konishi M, Yoshida M, Koshiyama M, et al. Primary treatment for 6 patients with carcinoma of presumably primary peritoneal origin. Adv Obstet Gynecol 50: 195-200, 1998 (in Japanese).

11) Sunami T, Kanemura $S$, Ohira $M$, You $T$. A case of peritoneal serous papillary adenocarcinoma. Jpn J Gastroenterol Surg 36: 1321-1326, 2003 (in Japanese).

12) Zissin R, Hertz M, Shapiro-Feinberg M, Bernheim J, Altaras M, Fishman A. Primary serous papillary carcinoma of the peritoneum: CT findings. Clin Radiol 56: 740-745, 2001.

13) Kebapci M, Yalcin OT, Dundar E, Ozalp SS, Kaya T. Computed tomography findings of primary serous papillary carcinoma of the peritoneum in women. Eur J Gynaecol Oncol 24: 552-556, 2003.

14) Chiou SY, Sheu MH, Wang JH, Chang CY. Peritoneal serous papillary carcinoma: a reappraisal of $\mathrm{CT}$ imaging features and literature review. Abdom Imaging 28: 815-819, 2003.

15) Whitley NO, Brenner DE, Antman KH, Grant D, Aisner J. CT of peritoneal mesothelioma: analysis of eight cases. Am J Roentgenol 138: 531-535, 1982.

16) Smith TR. Malignant peritoneal mesothelioma: marked variability of CT findings. Abdom Imaging 19: 27-29, 1994.

17) Cooper C, Jeffrey RB, Silverman PM, Federle MP, Chun GH. Computed tomography of omental pathology. J Comput Assist Tomogr 10: 62-66, 1986

18) Trauner M, Klimpfinger M, Stauber RE, Schreiber F, Stoger H, Krejs GJ. Laparoscopic diagnosis of peritoneal serous papillary carcinoma. Endoscopy 27: 337-341, 1995.

19) Ordonez NG. The immunohistochemical diagnosis of mesothelioma. Am J Surg Pathol 27: 1031-1051, 2003.

20) Oates J, Edwards C. HBME-1, MOC-31, WT1 and calretinin: an assessment of recently described markers for mesothelioma and adenocarcinoma. Histopathology 36: 341-347, 2000.

21) Gumurdulu D, Zeren EH, Cagle PT, et al. Specificity of MOC-31 and HBME-1 immunohistochemistry in the differential diagnosis of adenocarcinoma and malignant mesothelioma: a study on environmental malignant mesothelioma cases from Turkish villages. Pathol Oncol Res 8: 188-193, 2002.

22) Gonzalez-Lois C, Ballestin C, Sotelo MT, Lopez-Rios F, Garcia-Prats MD, Villena V. Combined use of novel epithelial (MOC-31) and mesothelial (HBME-1) immunohistochemical markers for optimal first line diagnostic distinction between mesothelioma and metastatic carcinoma in pleura. Histopathology 38: 528-534, 2001.

23) Attanoos RL, Webb R, Dojcinov SD, Gibbs AR. Value of mesothelial and epithelial antibodies in distinguishing diffuse peritoneal mesothelioma in females from serous papillary carcinoma of the ovary and peritoneum. Histopathology 40: 237-244, 2002.

24) Taus P, Petru E, Gucer F, Pickel H, Lahousen M. Primary serous papillary carcinoma of the peritoneum: a report of 18 patients. Eur J Gynaec Oncol 18: 171-172, 1997.

25) Ransom DT, Patel SR, Keeney GL, Malkasian GD, Edmonson JH. Papillary serous carcinoma of the peritoneum: a review of 33 cases treated with platin-based chemotherapy. Cancer 66: 1091-1094, 1990.

26) Ben-Baruch G, Sivan E, Moran O, Rizel S, Menczer J, Seidman DS. Primary peritoneal serous papillary carcinoma: a study of 25 cases and comparison with stage III-IV ovarian papillary serous carcinoma. Gynecol Oncol 60: 393-396, 1996.

27) Look M, Chang D, Sugarbaker PH. Long-term results of cytoreductive surgery for advanced and recurrent epithelial ovarian cancers and papillary serous carcinoma of the peritoneum. Int J Gynecol Cancer 13: 764-770, 2003. 